\title{
An Edge Detection Algorithm Based on Adaptive Threshold
}

\author{
Wei Jian Mo* \\ Xinhua College of Sun Yat-sen University, Guangzhou 510520,China \\ 472252720@qq.com
}

Keywords: Edge detection algorithm; Wavelet decomposition; Directional characteristics; Threshold.

\begin{abstract}
The threshold need artificial hypothesis in the existing edge detection algorithm. In response to this phenomenon, we propose an edge detection algorithm is based on adaptive threshold. Article algorithm was studying the characteristics of wavelet transform. And it was combined with the traditional edge detection algorithm to reset the threshold. So we can use this adaptive Threshold to search the edge signal. Experimental results show that the results consistent with the expected results. Article algorithm can kept a better edge detection effect under different condition. And it cans effective extract the edges of each frequency sub-band.
\end{abstract}

\section{Introduction}

The edge is one of the most fundamental features of image. It is the junction of the property area to another property area. It is the place where regional attribute mutations. So it has a wealth of information. Therefore, Edge image extraction having a key role in computer processing. Nowadays, have a lot of edge extraction operators [1-3]. For example: Roberts, Sobel, Canny, etc. It strengthened the processing of the image to a certain extent. But the disadvantage is susceptible to noise interference. In recent years, researchers proposed many new algorithms[4, 5]. For example: Edge detection algorithm base on wavelet Transform, Edge detection algorithm base on mathematical morphology, Edge detection algorithm base on energy guidelines, etc. These methods have made better results than traditional methods. However, there exists a great mount of computations. The computing speed is not acceptable for practice, either.

Focusing on the problem, we have research of the traditional edge detection algorithm and wavelet transform[6, 7].And proposed an edge detection algorithm based on adaptive threshold. It was redefines the computing and threshold of the image edge. High frequency sub-bands for different directions using different search window to get the wavelet coefficients. And adaptive adjustment of global threshold and local threshold to enhance the effect of edge detection. At the same time, In order to avoid the maximum positioning error in the threshold of the process. Article algorithm was according to the characteristics of edge gradient and gradient variance to improve threshold.

\section{Traditional edge detection algorithm based on wavelet}

By wavelet transform, we can get that $W_{\mathrm{f}}(a, b)=\frac{1}{\sqrt{C_{\psi}}} \frac{1}{\sqrt{|a|}} \int_{-\infty}^{+\infty} f(t) \bar{\psi}\left(\frac{t-b}{a}\right) d t$.

Therein $C_{\psi}=2 \pi \int_{-\infty}^{+\infty} \frac{|\psi(\omega)|^{2}}{|\omega|} \mathrm{d} \omega, \psi(\omega)$ is Fourier transform of $\psi(t)$, a、 $b$ is a stretching factor and 
shift factor on the image. Hypothesis $\theta(x, y)$ is smooth functions.

There is 2d wavelet coefficients calculation function: $\left\{\begin{array}{l}W_{2^{j}}^{1} f(x, y)=f * \psi_{2^{j}}^{1} f(x, y) \\ W_{2^{j}}^{2} f(x, y)=f * \psi_{2^{j}}^{2} f(x, y)\end{array}\right.$

Denoted by: $\quad W_{2^{j}} f(x, y)=\left[\begin{array}{l}W^{1}{ }^{j} f(x, y) \\ W^{2}{ }^{j} f(x, y)\end{array}\right]=2^{j}\left[\begin{array}{c}\frac{\partial}{\partial x} f \theta_{2^{j}}(x, y) \\ \frac{\partial}{\partial y} f \theta_{2^{j}}(x, y)\end{array}\right]=2^{j} \nabla\left(f \theta_{2^{j}}\right)(\mathrm{x}, \mathrm{y})$

When defining the scale $2^{j}$, Mold and argument expression function as follows

Mold: $M_{2^{j}} f(x, y)=\sqrt{\left|W_{2^{j}}^{1} f(x, y)\right|^{2}+\left|W_{2^{j}}^{2} f(x, y)\right|^{2}}$ (3) Argument: $\left.A_{2^{j}} f(x, y)=\arctan \frac{W_{2^{j}}^{2} f(x, y)}{W_{2^{j}}^{1} f(x, y)}\right)$

So membership function of $M_{2^{j}} f(x, y): U\left[M_{2^{j}} f(x, y)\right]=\left\{\begin{array}{c}\quad, M_{2^{j}} f(x, y) \geq \beta \\ \left(1+\left(\operatorname{Max}-M_{2^{j}} f(x, y)\right)^{2}\right)^{-1}, \alpha<M_{2^{j}} f(x, y)<\beta \\ 0 \quad, M_{2^{j}} f(x, y) \leq \alpha\end{array}\right.$

Therein Max is the maximum of the gradient direction. $\alpha$ and $\beta$ are changed depending on the image [8]; And it was using the join- meet operation to get the information for each scale. The functional operation is as follows.

meet: $\quad\left(U_{2^{\mathrm{i}}} f(x, y)\right) \cap\left(U_{2^{\mathrm{j}}} f(x, y),(i \neq j, i, j \in s) ; \quad\right.$ join: $\quad U f(x, y)=U\left[\left(U_{2^{\mathrm{i}}} f(x, y)\right) \cap\left(U_{2^{\mathrm{j}}} f(x, y)\right)\right]$

Therein $i \neq j, i, j \in S$; In the end, Through using the $U f(x, y)$, And combined with the

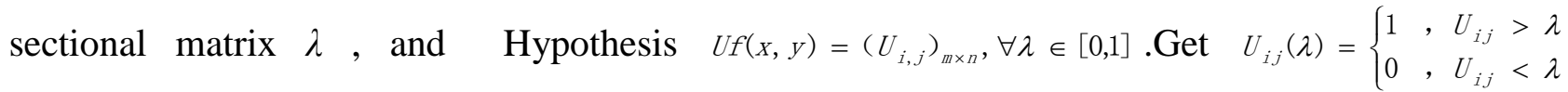

\section{Article algorithm}

Calculation of pixel values. For directional characteristic of wavelet decomposition, article algorithm was use a sliding type of edge detection operator. And article algorithm was using the gray value of the pixel point to multiply by the value of the edge detection operator. It was to instead of the gray value of pixel. And article algorithm was by moving the sliding window for re-assignment each pixel.

Figure. 1 is $3 \times 3$ sliding detection window. These nine pixels are denoted as $P_{i}, i=1,2, \cdots, 9$. when calculation of pixel values, we should find in the sliding detection window[9].

\begin{tabular}{|c|c|c|}
\hline P1(x-1,y+1) & P2(x,y+1) & P3(x+1,y+1) \\
\hline P4(x-1,y) & P5(x,y) & P6(x+1,y) \\
\hline P7(x-1,y-1) & P8(x,y-1) & P9(x+1,y-1) \\
\hline
\end{tabular}

Figure. $13 \times 3$ sliding detection window

\begin{tabular}{|c|c|c|}
\hline 0 & 0 & 0 \\
\hline-1 & 4 & -1 \\
\hline 0 & 0 & 0 \\
\hline
\end{tabular}

\begin{tabular}{|l|l|l|}
\hline 0 & -1 & 0 \\
\hline 0 & 4 & 0 \\
\hline 0 & -1 & 0 \\
\hline
\end{tabular}

\begin{tabular}{|c|c|c|}
\hline-1 & 0 & -1 \\
\hline 0 & 4 & 0 \\
\hline-1 & 0 & -1 \\
\hline
\end{tabular}

(a) horizontal direction (b) vertical direction (c) diagonal directions

Figure. 2 edge detection template of this paper 
When to search for the pixels, sliding detection window was automatic adjustment based on Sobel edge detection operator. Final block diagram shown in Figure 2. It can using different templates to calculated different directions of the sub-band.

Selected threshold. If we were used to determine the maximum threshold for each direction, must be leads the probems that discrete of edge signal and positioning error[10]. If combined signals of each direction of edge at this time, must be can not get the ideal edge signal. Therefore, article algorithm was redefine the threshold. It was according to the characteristics of the different sub-band coefficient.as follows 。

Local threshold. Here we only consider about one threshold. It was The optimal value of each high-frequency sub-bands.Its expression functions are as follows:

$$
\tau=H_{\max }+S\left(M_{2^{\mathrm{j}}} f(x, y)\right)
$$

Therein $H_{\max }$ Is the best local edge gradient, $S\left(M_{2^{j}} f(x, y)\right)$ Is a partial edge gradient variance.

$H_{\max }$ function expression: $H_{\max }=\operatorname{MAX}\left(M_{2^{\mathrm{j}}} f(x, y)\right)$

$$
S\left(M_{2^{j^{j}}} f(x, y)\right) \text { function expression: } S\left(M_{2^{\mathrm{j}}} f(x, y)\right)=\sum_{i=1}^{n} \frac{\left(M_{2^{\mathrm{j}}} f(x, y)-\overline{M_{2^{\mathrm{j}}} f(x, y)}\right)^{2}}{n}
$$

It was benefit our accord the size of the signal strength to adjust in the case of local optima.

Global threshold. Article algorithm was according to the characteristics of global optimum.

Hypothesis $\tau_{H}$ and $\tau_{L}$ were the highest and lowest threshold. Its expression functions are as follows:

$$
\tau_{H}=(1-\beta) \tau_{\max }+\beta \tau_{\max } \quad(7) ; \quad \tau_{L}=(1-\beta) \tau_{\min }+\beta \tau_{\min }
$$

Therein $0<\beta<1$ is the adjust rate of global optimal threshold。In the actual image edge detection, $\beta$ is adjusted according to the size of the sub-graph.

Processing edge signal again. According to the size of wavelet modulus values, we can distinguish edge signal and a background signal in the image. And we have according to the membership function characteristics of attenuating operation in the edge signal. Let the hard-over edge signal to weakening, and let the weak edge to enhance. Set up a new membership function. Its expression functions are as follows:

When $M_{2^{\mathrm{j}}} f(x, y) \geq \tau_{H}$ or $\left.M_{2^{\mathrm{j}}} f(x, y)>\tau, \quad \lambda_{i}=\frac{1}{2}\left(M_{2^{\mathrm{j}}} f(x, y)\right) / \tau\right)$

When $M_{2^{\mathrm{j}}} f(x, y) \leq \tau_{L}$ or $M_{2^{\mathrm{j}}} f(x, y)<\tau, \quad \lambda_{i}=1-\frac{1}{2}\left(\frac{\max \left[M_{2^{\mathrm{j}}} f(x, y)\right]-M_{2^{\mathrm{j}}} f(x, y)}{\max \left[M_{2^{\mathrm{j}}} f(x, y)\right]-\tau}\right)$

On the fuzzy feature plane, ${ }^{\lambda_{i}}$ was computation with Iterative enhancement. Its expression functions are as follows: $\lambda_{i}^{\prime}=T_{r}\left(\lambda_{i}\right)=T_{1}\left(T_{r-1}\left(\lambda_{i}\right)\right), \mathrm{r}$ is the number of iterations, Its expression 
functions are as follows: $T_{r}\left(\lambda_{i}\right)=\left\{\begin{array}{cc}2 \lambda_{i}^{2} & 0 \leq \lambda_{i} \leq 0.5 \\ 1-2\left(1-\lambda_{i}^{2}\right) & 0.5<\lambda_{i} \leq 1\end{array}\right.$

At the end, we get the best edge signal that was using it to Inverse transformation. Its expression functions are as follows: ${ }^{\left[M_{2^{j}} f(x, y)\right]^{\prime}=G^{-1}\left(\lambda_{i}^{\prime}\right)}$

\section{Simulation experiments}

The simulation tool used Matlab 7.10.0. And in order to detect the feasibility of article algorithm, we was select the typical images to experiments that according to different edge. By observing the simulation to test the feasibility of the algorithm. Table 1was classification table of the image edge.

Table 1 Classification table of the image edge

\begin{tabular}{ccc}
\hline edge & characteristic & Image Name \\
\hline greater than 0.6 & Pure textures & wall、 beans bark \\
$0.4 \sim 0.6$ & textures / Edge & baboon、goldhill、plant \\
$0.2 \sim 0.1$ & textures / Edge / Smooth & lena、pepper、boat \\
$0.1 \sim 0.2$ & Edge / Smooth & circle、pillsetc、gray \\
\hline
\end{tabular}

Article algorithm was select the typical images to experiments. It can verify the feasibility of this algorithm. Which according to the edges of high to low: beans, baboon, pepper, pillsetc. The simulation is shown below.
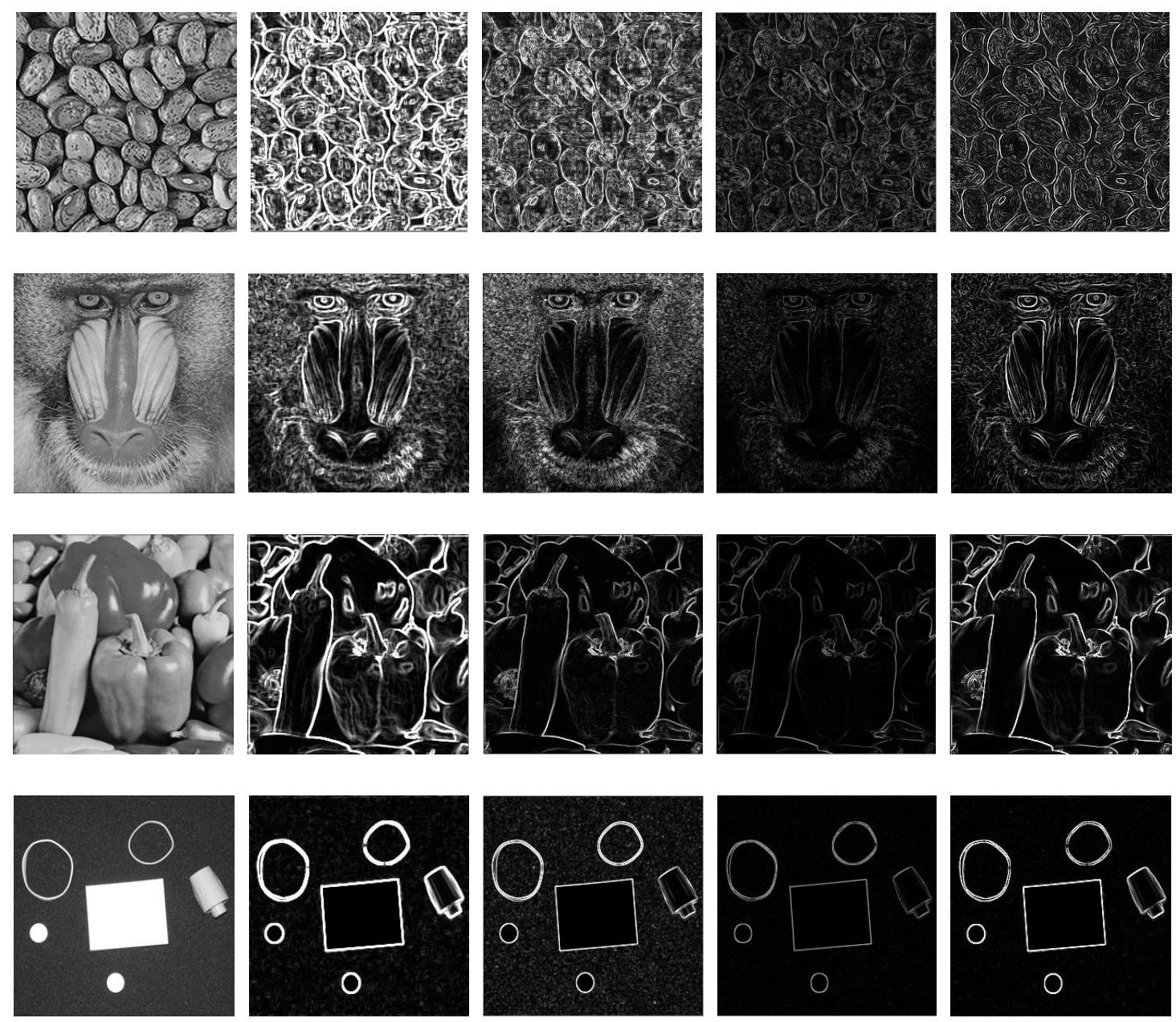

(a) Original image (b) The first layer of synthetic edge (c) The second layer of synthetic edge (d) The third layer of synthetic edge (e) re-processed image

Figure. 3 Simulation map of articles algorithm 
Through the observation found that the edge information was layer by layer to weakened. After different image processing, we can see that edge detail is gradually clear. After the re-processed, we can get the clearer edge. Although when we processing the beans image, edge information was affected by the texture signal. But it was kept within a reasonable range. It was proved that algorithm results was consistent with the expected effect.

\section{Summary}

Proposed an Edge detection algorithm based on adaptive threshold. Different directions of the high-frequency sub-bands were using a targeted search window. It is beneficial to improve the edge extraction effect. In the course of the threshold, articles algorithm was introduction edge detection of the Threshold. So we was using it to redefined the global and local variables. Experimental results show that articles algorithm can efficient extraction of edge single in each high-frequency sub-bands. And there was have a strong edge extraction effect in images of different complexity.

\section{References}

[1] Huang BW; Yuan J. A New Adaptive Threshold Image-Denoising Method Based on Edge Detection [J]. IAES TELKOMNIKA Indonesian Journal of Electrical Engineering. 2014, 12(5): 3509-3514.

[2] Liu B, Zhang ZH, Liu XZ, Yu WX. Edge Extraction for Polarimetric SAR Images Using Degenerate Filter With Weighted Maximum Likelihood Estimation [J]. IEEE GEOSCIENCE AND REMOTE SENSING LETTERS. 2014,11(12): 2140-2144.

[3] Chavez-Roman H, Ponomaryov V. Super Resolution Image Generation Using Wavelet Domain Interpolation With Edge Extraction via a Sparse Representation [J]. IEEE GEOSCIENCE AND REMOTE SENSING LETTERS. 2014,11(10): 1777-1781

[4] Baselice F, Ferraioli G, Reale D. Edge Detection Using Real and Imaginary Decomposition of SAR Data [J]. IEEE TRANSACTIONS ON GEOSCIENCE AND REMOTE SENSING. 2014,52(7): 3833-3842.

[5] Chen W, Tian QC, Liu J, Wang QP. Nonlocal low-rank matrix completion for image interpolation using edge detection and neural network [J]. SIGNAL IMAGE AND VIDEO PROCESSING. 2014,8(4): 657-663.

[6] Lahmiri S. IMPROVING FORECASTING ACCURACY OF THE S\&P500 INTRA-DAY PRICE DIRECTION USING BOTH WAVELET LOW AND HIGH FREQUENCY COEFFICIENTS [J]. FLUCTUATION AND NOISE LETTERS. 2014,13(1):251-262

[7] Hong XT, Shi XW, Qian YX. Optical Correlation Recognition Based on Edge Detection of Wavelet Transform [J]. Laser \& Optoelectronics Progress. 2014,51(4):1023-1035.

[8] Zhang ZS, Lv HX, Zhou QB, Zhang HQ. Reconstructing Sparse Signals from Dyadic Wavelet Transform Modulus Maxima[J].CIRCUITS SYSTEMS AND SIGNAL PROCESSING. 2014,33(8): 2667-2674.

[9] Li YR, Xu HN. Performance Comparison of Contourlet Transform and Directional-Wavelet Transform [J]. Electronics Optics \& Control. 2013,20(8): 79-83.

[10]Fan YJ, Wu XH, Luo DS. A modified image edge detection algorithm based on wavelet transform [J]. Sichuan Daxue Xuebao (Ziran Kexueban). 2012,49(6): 1264-1268. 